Case Report

\title{
Prolonged and Resistant Cutaneous Infection in Newly Transplanted Renal Patient
}

Sofia Oliveira Correia ${ }^{1,}{ }^{*}$, Catarina Eusébio ${ }^{2}$, Ana Sofia Cipriano ${ }^{3}$, Virgínia Lopes ${ }^{4}$, Ramon Vizcaíno ${ }^{5}$, António Cabrita ${ }^{1}$, Manuela Almeida ${ }^{1}$, Sofia Pedroso ${ }^{1}$, Leonidio Dias ${ }^{1}$, La Salete Martins ${ }^{1}$, António Castro Henriques ${ }^{1}$

1. Nephrology and transplant unit, Centro Hospitalar do Porto, Portugal; E-Mails: soacorreia@gmail.com; acabrita.nefrologia@chporto.min-saude.pt; manuela.almeida10@gmail.com; sofialpedroso@gmail.com; leonidiodias@sapo.pt; lasalet@gmail.com; antonioach@hotmail.com

2. Nephrology department, Centro Hospitalar de Trás-os-Montes e Alto Douto, Portugal; E-Mail: catarina.ip.eusebio@gmail.com

3. Infectious diseases, Centro Hospitalar do Porto, Portugal; E-Mail: acipriano88@gmail.com

4. Clinical Microbiology Department, Centro Hospitalar do Porto, Portugal; E-Mail: gilopes61@gmail.com

5. Department of Pathological Anatomy, Centro Hospitalar do Porto, Portugal; E-Mail: jrvizcaino@gmail.com

* Correspondence: Sofia Oliveira Correia; E-Mail: soacorreia@gmail.com

Academic Editor: Yasuhiko Sugawara

OBM Transplantation

2019, volume 3, issue 1

doi:10.21926/obm.transplant.1901057
Received: October 18, 2018

Accepted: February 01, 2019

Published: March 07, 2019

\begin{abstract}
Mucormycosis is an angioinvasive and potentially fatal fungal infection caused by ubiquitous filamentous fungi. Cutaneous mucormycosis is an emerging infection and potentially lethal. Our aim was to review the literature on cutaneous mucormycosis. We present a case of a renal transplant patient with a surgical wound infection 20 days after kidney transplant. This case is an example on how early diagnosis is crucial and, since culture is less sensitive, microscopic evaluation of the characteristic broad hyphae is the most crucial diagnostic tool.
\end{abstract}

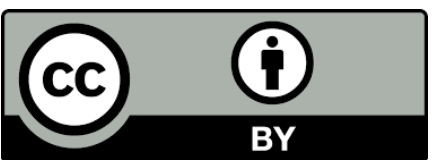

(C) 2019 by the author. This is an open access article distributed under the conditions of the Creative Commons by Attribution License, which permits unrestricted use, distribution, and reproduction in any medium or format, provided the original work is correctly cited. 


\section{Keywords}

Mucormycosis; solid organ transplant

\section{Introduction}

Mucorales are ubiquitous saprophytic fungi which exist widely in nature. The agents of mucormycosis represent a heterogeneous group of organisms, which encompass the genera Rhizopus, Mucor, Cunninghamella, Rhizomucor, Lichtheimia, and others.

Mucormycosis can manifest in different clinical presentations and locations: rhinoorbitocerebral, cutaneous, pulmonary, disseminated, gastrointestinal and other rare forms. Mucormycosis accounts for approximately $2 \%$ of fungal infections in solid organ transplant recipients [1].

Diagnosis is difficult and its delay can jeopardize prompt treatment. In order to prevent fungi dissemination and improve survival, anti-fungal agents, surgery, and control of the underlying condition contributing to the immunosuppressive states are strongly recommended. Depending on the extent and location of the disease, mortality rates can be up to $48 \%$. We encountered a case of cutaneous mucormycosis in a transplanted renal patient.

This article addresses the clinical features, diagnosis and treatment supported by a review of the recent literature.

\section{Clinical Case}

A 62-year-old male patient with chronic kidney disease secondary to IgA Nephropathy on hemodialysis since 1999, underwent renal transplantation in 2001, had immediate renal graft function and had no episodes of rejection.

Due to chronic graft dysfunction he re-initiated hemodialysis in 2009. There is no other relevant personal history beyond hypertension and secondary hyperparathyroidism. There was no recent history of travel or risk epidemiological context and he lives in an urban area.

In 2017, he underwent re-transplantation. Anti-thymocyte globulin (ATG) was given as an induction immunosuppressive agent. The use of mycophenolate mofetil (MMF), tacrolimus and prednisolone was later given due to the presence of anti-donor antibodies that placed him at a high immunological risk. He had delayed graft function for five days, requiring dialysis. He was discharged with serum creatinine of $3.56 \mathrm{mg} / \mathrm{dL}$. In the first weeks after transplantation, his minimal creatinine reached was $2.9 \mathrm{mg} / \mathrm{dL}$, then graft biopsy was proposed.

The surgical wound became red with swollen skin, and hot and tender to the touch. It was treated initially with amoxicillin for two weeks but evolved to a bump that appeared below the skin's surface. Renal graft biopsy was postponed for this reason. An ultrasound was performed and revealed a collection of approximately $7.3 \times 1.2 \times 3.5 \mathrm{~cm}$ in the abdominal wall. Fluid from the abscess were then collected for microbiological study. The bacterial culture from the fluid were sterile. Physical evaluation was otherwise normal. A C-reactive protein elevation $(14,47 \mathrm{mg} / \mathrm{L}$, normal range: $<5 \mathrm{mg} / \mathrm{L}$ ) and mild pancytopenia was identified in the blood tests. He was discharged with flucloxacillin as treatment. 
The lesion evolved to a painful, indurated, confluent, suppurative and embossed area of cellulitis (Figure 1). New drainage was performed to obtain a sample for microbiological study, including fungal culture. After 2 weeks of incubation, fungal culture revealed the presence of Lichtheimia corymbifera (Figure 2).
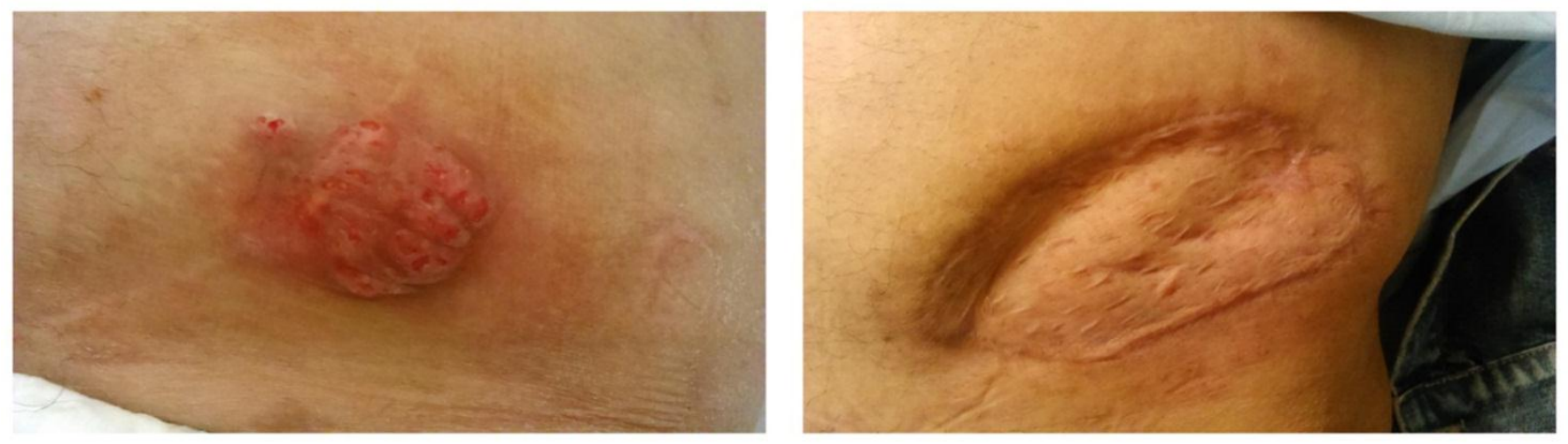

Figure 1 Cutaneous mucormycosis: Initial injury on the left and plasty with skin graft on the right.
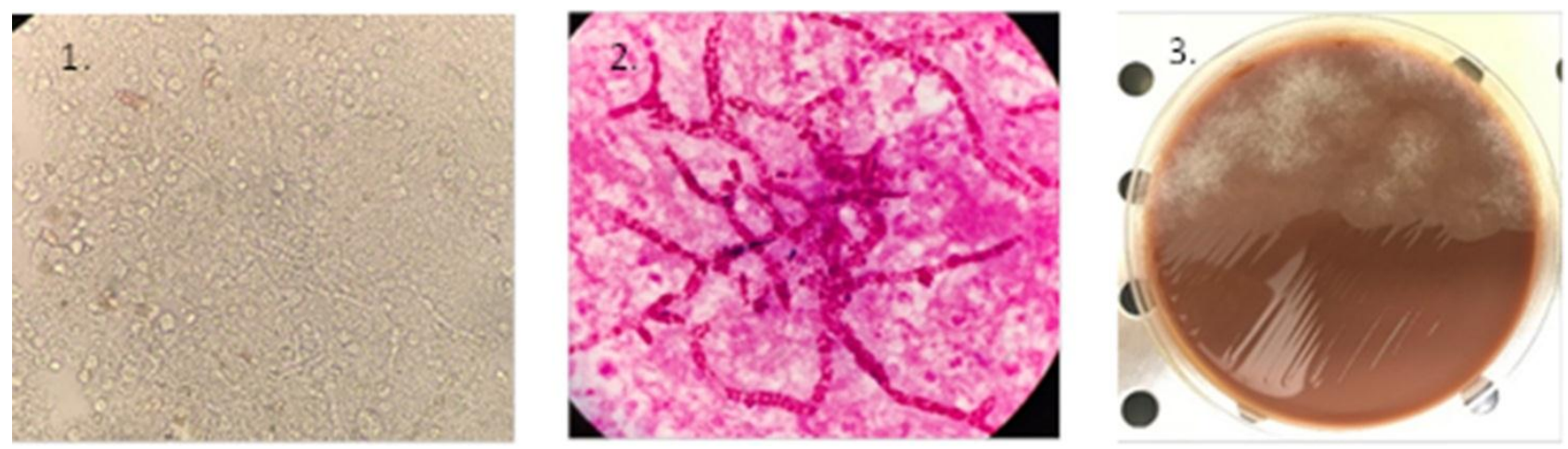

Figure 2 Direct microscopy of the surgical wound suppuration. Clarification with potassium hydroxide revealed numerous non-septate hyphae with branching at 900 (1) also visualized by gram stain (2); Culture in chocolate agar - Fungal colonies of coenocytic fungi (3).

Computed tomography $(C T)$ of the thorax, abdomen and pelvis did not reveal lesions suggestive of dissemination. Steroid therapy was reduced to prednisolone $5 \mathrm{mg}$ and MMF was suspended.

Amphotericin B (5 mg/kg/day) was given for 40 days in association with posaconazole (delayed release, $300 \mathrm{mg}$ id once daily) that was suspended later for a total of 53 days of treatment. Surgical removal of the lesion was performed early after admission. Later, he underwent curettage and plasty with skin graft that evolved to total cicatrization of the scar (Figure 1).

There was no recovery of renal function. His immunosuppression was maintained with prednisolone and tacrolimus with dose reduction while receiving posaconazole, for levels within the goal $(6-8 \mathrm{ng} / \mathrm{ml})$. No kidney biopsy was made. One year later, creatinine is $3.6 \mathrm{mg} / \mathrm{dL}$. He had no other infectious complications. 


\section{Discussion}

Among solid organ transplant recipients, risk factors for mucormycosis include renal failure, diabetes, prior anti-fungal therapy, prolonged and profound immunodeficiency, breaks in skin integrity, and chronic respiratory disease [2, 3]. Mucormycosis accounts for $2 \%$ of fungal infections in solid organ transplant recipients [3]. These organisms are encountered worldwide.

Although the correct diagnosis is often difficult, an early identification is essential for patient survival. The diagnosis of mucormycosis relies upon the identification of organisms in tissue by histopathology with culture confirmation. In this context, good quality representative clinical samples as well as rapid laboratory response are critical to the diagnosis.

Since culture is less sensitive, microscopic demonstration of the characteristic broad hyphae in clinical specimens is the most crucial diagnostic tool and should never be ruled out. Specific fungal stains should be included [4].

Infection of skin and soft tissues with zygomycetes results from direct inoculation of the spores into the dermis or inhalation, for this reason, prevention is key. Sites of medical or surgical interventions are the most frequent site of infection [3]. Patients should be instructed to avoid visiting construction sites and farms, manipulating air-conditioning filters, and contact with sewage or decaying material. Also, all fungal isolates from a donor should be reported [3].

There are a few case reports of cutaneous mucormycosis. A review of 929 cases of Zygomycosis had cutaneous involvement in 19\% [5]. Different presentations has been described: ecthyma-like lesions and black necrotic cellulitis [6], purulent discharging sinus at the surgical incision site [7] and erythema-nodosum-like lesions [8].

Rapid initial care and treatment is important to prevent fungi dissemination and improve survival. The treatment strategy can be based on lipid formulations of amphotericin B (L-AmB 5$10 \mathrm{mg} / \mathrm{Kg} /$ day) as first-line drug treatment for mucormycosis, with amphotericin-B-echinocandin combinations listed as an option for salvage therapy [9], or combination therapy up front [10]. L$A m B$ doses of $10 \mathrm{mg} / \mathrm{kg} /$ day are suggested for infections involving the central nervous system.

Caspofungin, anidulafungin and micafungin have no efficacy against agents of mucormycosis as single agents when tested by standard techniques in vitro.

Posaconazole (200 mg four times daily or $400 \mathrm{mg}$ twice daily with solution or $300 \mathrm{mg}$ once daily for tablets) or isavuconazole can be added in recalcitrant cases [10,11]. With clinical improvement, treatment can be switched to posaconazole monotherapy. Fluconazole and voriconazole have no meaningful activity against agents of mucormycosis in vitro. Based on expert opinions and existing data, no other azoles, except posaconazole, are recommended in the treatment of mucormycosis.

Therapeutic drug-monitoring is essential: monitor for Q-T interval prolongation, drug interactions, hepatotoxicity and neuropsychiatric side effects with azoles and renal toxicity with amphotericin B products [3]. Dose adjustments can be guided by laboratory drug measures (target voriconazole: levels between $1.5-4.5 \mathrm{lg} / \mathrm{mL}$ and posaconazole: at least $1 \mu \mathrm{g} / \mathrm{mL}$ and ideally more than $2 \mu \mathrm{g} / \mathrm{mL}$ ) even though these levels are based on very limited data [3, 11].

Immunosuppression has to be reduced [3]. Stopping steroids and anti-metabolites is advisable and reduction of $\mathrm{CNI}$ or mTOR inhibitor doses should be considered [10]. Surgical debridement (sometimes repeatedly) of the affected areas should be performed whenever feasible [3]. Surgical debridement is considered the standard of treatment of cutaneous and surrounding tissue mucormycosis and has been found to improve outcome [9]. 
The combination of caspofungin with calcineurin inhibitors (CNI) and sirolimus (both have intrinsic anti-fungal activity) was described to be synergistic for the agents of mucormycosis [12]. Another non-traditional combination that can be considered is iron chelators and statins [10]. Hyperbaric oxygen as adjunctive therapy can increase tissue concentration of oxygen and may increase neutrophil anti-fungal activity. It has been used in diabetic patients with rhino-orbitocerebral disease but there is not enough data to support its use routinely. The use of granulocyte colony-stimulating factor (G-CSF), granulocyte- macrophage colony-stimulating factor (GM-CSF), and interferon- $\gamma$ (IFN- $\gamma$ ) as adjunctive treatment beyond the setting of granulocytopenia in nonneutropenic patients was also tested in some case reports. There is insufficient data to recommend its use in non-neutropenic patients [9].

There are no data on treatment duration thus, therapy should be continued until all clinical and radiographic signs of infection have resolved and at least for 6 weeks. The outcome depends on several factors, including the site of infection, the immune status of the host and the use of surgery or other adjunctive treatments. A prospective randomized clinical trial would be ideal to establish the best treatment approach. The rarity of this condition states that a large international collaborative effort is needed.

\section{Conclusions}

Primary cutaneous mucormycosis is an emerging fungal infection and should be considered when skin /soft tissue infection is refractory to anti-microbial therapy. Early diagnosis, surgical excision and appropriate anti-fungal therapy are important to improve outcomes.

\section{Author Contributions}

All authors analyzed and interpreted the patient data and were responsible for the diagnostic assessment. SC was a major contributor in writing the manuscript. All authors read and approved the final manuscript.

\section{Funding}

No specific funding was obtained for this study.

\section{Competing Interests}

The authors have declared that no competing interests exist.

\section{References}

1. Pappas PG, Alexander BD, Andes DR, Hadley S, Kauffman CA, Freifeld A, et al. Invasive fungal infections among organ transplant recipients: results of the Transplant-Associated Infection Surveillance Network (TRANSNET). Clin Infect Dis. 2010; 50: 1101-1111.

2. Singh N, Aguado JM, Bonatti H, Forrest G, Gupta KL, Safdar N, et al. Zygomycosis in solid organ transplant recipients: a prospective, matched case-control study to assess risks for disease and outcome. J Infect Dis. 2009; 200: 1002-1011. 
3. Huprikar S, Shoham S. Emerging fungal infections in solid organ transplantation. Am J Transplant. 2013; 13: 262-271.

4. Ruhnke M, Behre G, Buchheidt D, Christopeit M, Hamprecht A, Heinz W, et al. Diagnosis of invasive fungal diseases in haematology and oncology: 2018 Update of the recommendations of the infectious diseases working party of the german society for hematology and medical oncology (AGIHO). Mycoses. 2018; 61: 796-813.

5. Roden MM, Zaoutis TE, Buchanan WL, Knudsen TA, Sarkisova TA, Schaufele RL, et al. Epidemiology and outcome of zygomycosis: A review of 929 reported cases. Clin Infect Dis. 2005; 41: 634-653.

6. Adriaenssens K, Jorens PG, Meuleman L, Jeuris W, Lambert J. A black necrotic skin lesion in an immunocompromised patient. Diagnosis: cutaneous mucormycosis. Arch Dermatol. 2000; 136: 1165-1170.

7. Parikh A, Tuli A, Sridhar FK, Mammen K. Mucormycosis in the post renal transplant surgical scar. Indian J Transplant. 2015; 9: 116-118.

8. Nouri-majalan N, Moghimi M. Skin mucormycosis presenting as an erythema-nodosum-like rash in a renal transplant recipient : A case report. J Med Case Rep. 2008; 4: 2-5.

9. Skiada A, Lanternier F, Groll AH, Pagano L, Zimmerli S, Herbrecht R, et al. Diagnosis and treatment of mucormycosis in patients with hematological malignancies: Guidelines from the 3rd European Conference on Infections in Leukemia (ECIL 3). Haematologica. 2013; 98: 492504.

10. Haidar G, Singh N. How we approach combination antifungal therapy for invasive aspergillosis and mucormycosis in transplant recipients. Transplantation. 2018; 102: 1815-1823.

11. Danion F, Aguilar C, Catherinot E, Alanio A, Dewolf S, Lortholary O, et al. Mucormycosis: New developments into a persistently devastating infection. Semin Respir Crit Care Med. 2015; 36: 692-705.

12. Thakur M, Revankar SG. In vitro interaction of caspofungin and immunosuppressives against agents of mucormycosis. J Antimicrob Chemother. 2011; 66: 2312-2314.

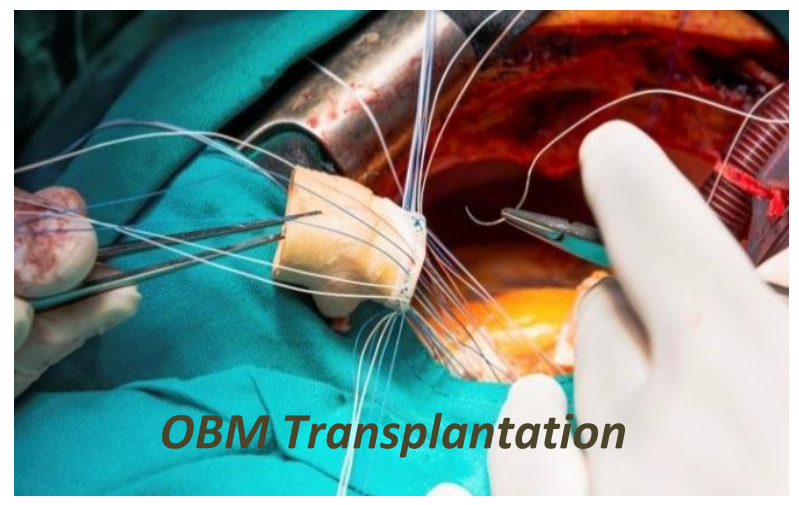

Enjoy OBM Transplantation by:

1. Submitting a manuscript

2. Joining in volunteer reviewer bank

3. Joining Editorial Board

4. Guest editing a special issue

For more details, please visit:

http://www.lidsen.com/journals/transplantation 